\title{
Congenital uterovaginal abnormalities, it's embryogenesis, surgical management and clinical implications
}

\author{
Krishna Gopal Paul, MS'1, Kalyan Chakravarthi Kosuri, MSc, PhD², Hasam Bayad, MD³ \\ Departments of ${ }^{1}$ Obstetrics and Gynaecology, ${ }^{2}$ Anatomy, Varun Arjun Medical College and Rohilkhand Hospital, Banthara, India; ${ }^{3}$ Department of \\ Obstetrics and Gynaecology, Princess Marine Hospital, Gaborone, Botswana
}

\section{Objective}

Congenital Mullerian duct malformations are a challenging group of conditions for surgeons and need surgical experience and skill. Accordingly, the aim of this study is to present the diagnosis, surgical management, and clinical implications of congenital uterovaginal abnormalities.

\section{Methods}

Between 1980 and 2015, 8 patients with congenital uterovaginal abnormalities were diagnosed. In one patient a unique case of an unusual horseshoe shaped double uterus communicating via a transverse canal along with agenesis of the cervix and vagina was noted, and utero-vaginal agenesis was diagnosed in 6 patients. Complete androgen insensitivity syndrome with its female phenotype associated with bilateral testicular tissue in the inguinal canal with an accompanying absence of the ovaries, uterus, uterine tubes, vagina, and an imperforate hymen, was diagnosed in one patient. Clinical examination of all the patients revealed well-developed secondary sexual characteristics. A modified McIndoe vaginoplasty procedure was the surgical treatment common to all patients to treat vaginal agenesis. The surgery was performed by a consultant (Dr. K.G. Paul) using the standardized surgical technique.

\section{Results}

An unusual Mullerian duct anomaly, uterus bicornisacollis, was successfully corrected by uteroplasty and a new cervix was constructed. Complete vaginal agenesis was corrected by a modified McIndoe vaginoplasty technique. None of the patients had any significant post-operative complications.

\section{Conclusion}

Knowledge of congenital uterovaginal abnormalities diagnosed in this study is essential for surgeons, clinical anatomists, radiologists, and morphologists who may increase the success of their diagnostic evaluations and surgical approaches to the region.

Keywords: Androgen insensitivity syndrome; Infertility; Vaginal agenesis; Wolffian duct

\section{Introduction}

The reproductive system or genital system is a system of sex organs within an organism involved in producing offspring. In men, the genital system includes the prostate, testes, and penis. In women, it includes the ovaries, fallopian tubes, uterus, and vagina. A basic understanding of the embryology of the reproductive tract is essential to restore reproductive and sexual functioning and normalize genital anatomy whenever possible.
Received: 2020.02.24. Revised: 2020.04.11. Accepted: 2020.05.05. Corresponding author: Kalyan Chakravarthi Kosuri, MSc, PhD Department of Anatomy, Varun Arjun Medical College and Rohilkhand Hospital, Lucknow Rd, District Shahjahanpur, Banthara 242307, India

E-mail: kalyankosuric@gmail.com https://orcid.org/0000-0001-7187-8687

Articles published in Obstet Gynecol Sci are open-access, distributed under the terms of the Creative Commons Attribution Non-Commercial License (http://creativecommons. org/licenses/by-nc/3.0/) which permits unrestricted non-commercial use, distribution, and reproduction in any medium, provided the original work is properly cited.

Copyright $\odot 2020$ Korean Society of Obstetrics and Gynecology 


\title{
Obstetrics \& Gynecology Science
}

\author{
Vol. 63, No. 5, 2020
}

The vagina is the elastic, muscular part of the female genital tract, which extends from the vulva to the cervix. In humans, the vagina receives sperms during sexual intercourse, is the channel for the birth canal during the birth process, and also functions as an excretory channel for menstrual flow. Congenital vaginal agenesis is a rare malformation estimated to occur among 1 in 4,000-5,000 female births. The Mclndoe technique was first described in 1938 by Bainster and Mclndoe as a surgical procedure that improves quality of life and sexual satisfaction and provides a functional vagina with minimal complications for most patients with vaginal agenesis. Congenital and acquired genital anomalies in the adolescent population may lead to difficulties in carrying a pregnancy to term, infertility, and recurrent pregnancy loss, which lead to a great amount of social inhibition and psychological distress for the affected individuals. Such abnormalities are a challenging group of conditions for the reproductive surgeon and need surgical experience and skill. They also require a basic knowledge of the embryology of the reproductive tract to increase the success of diagnostic evaluations and surgical approaches to the region. Accordingly, the aim of this study was to evaluate congenital uterovaginal abnormalities, and their embryogenesis, surgical management, and clinical implications.

\section{Materials and methods}

Between 1980 and 2015, 8 patients with congenital uterovaginal malformation were diagnosed in our clinics.

\section{Case I: congenital uterine malformation with vaginal agenesis}

A 20-year-old unmarried woman presenting with primary amenorrhea was admitted to the Gynaecology Clinic of Princess Marine Hospital, Gaborone, Botswana. A diagnostic laparoscopy procedure revealed a double uterus, with communication via a transverse canal, along with agenesis of the cervix, vagina, and external vaginal opening (hymen). The urethral orifice was normal and the patient had welldeveloped secondary sexual characteristics.

Surgical correction (uteroplasty) of a uterine malformation (Supplementary Fig. 1): a lower abdominal transverse incision was given and the abdomen was exposed; each medial wall of the uterus was cut from above with a downward trajec- tory until the endometrial cavity was entered. Each uterine cavity was then opened by separating the uterus into anterior and posterior walls. Once the endometrial cavities of both uteri were exposed, the uterine walls were then reapproximated and sutured together with synthetic absorbable sutures, and both uterine cavities were converted into a single full space. The lower end of the uterus was then opened and the surgeons created a cervix. The newly created cervix was supported by a small plastic tube that was removed after 6 weeks during Mclndoe vaginoplasty. Six weeks after the uteroplasty, McIndoe vaginoplasty was done to treat vaginal agenesis (Supplementary Fig. 1).

\section{Case II: utero-vaginal agenesis}

Six unmarried woman (age 19-23 years) presented with primary amenorrhea. Diagnostic laparoscopy procedures revealed the presence of well-developed ovaries with an absence of a vagina, hymen, uterus, and uterine tubes. The urethral orifice was normal with well-developed secondary sexual characteristics. In all 3 patients, McIndoe vaginoplasty was performed to treat vaginal agenesis. Out of 6 patients, one was operated at INHS Asvini, Mumbai between 1980 and 1983, 3 patients were operated at 166 Military Hospital, Jammu between 1993 and 1995, and 2 patients were operated at Command Hospital, Calcutta during between 2000 and 2003(Supplementary Fig. 2).

\section{Case III: complete androgen insensitivity syndrome}

A 20-year-old unmarried woman presenting with primary amenorrhea was admitted to the Gynaecology Department at the 166 Military Hospital, Jammu. A diagnostic laparoscopy procedure revealed the presence of bilateral testicular tissue (testicular feminization with its female phenotype) in the inguinal canal with an absence of ovaries, uterus, uterine tubes, and vagina, and an imperforate hymen. The urethral orifice and the external genitalia were normal. With the help of a geneticist, the karyotype was mapped and revealed a female phenotype, 46, XY. The testes were removed in order to avoid a risk of malignancy (Supplementary Fig. 3).

\section{Modified McIndoe vaginoplasty}

To treat vaginal agenesis, 7 patients (casel and casell) aged between 19 to 23 years underwent McIndoe vaginoplasty. The patients were operated on under general anaesthesia and placed in a lithotomic position with urinary catheteriza- 


\section{Obstetrics \& Gynecology Science}

Krishna Gopal Paul, et al. Congenital uterovaginal abnormalities

tion (Supplementary Fig. 4). The surgery was performed by consultant Dr. K.G. Paul using the standardized surgical technique. In this operation, the labia are retracted with Allis clamps, a transverse incision is made in the epithelium, and blunt finger dissection is then carried out to create a new vaginal space or canal (optimum vaginal length of 10-12 $\mathrm{cm}$ ) between the rectum and bladder. Two proposed paddleshaped full-thickness skin grafts approximately $10 \mathrm{~cm}$ in length and $6 \mathrm{~cm}$ in width over the inguinal ligaments from the anterior superior iliac spine to the pubic tubercles were harvested from the patient (Supplementary Fig. 5). After harvesting the full-thickness skin graft, to close the wound, the skin is stretched and sutured using a synthetic absorbable suture (Supplementary Fig. 5). These full-thickness skin grafts allow for sufficient penetration of the transudation nutrients from the bed of the graft that are necessary for nutrition during the first 72 hours until microcapillary growth has been completed, which reduces postoperative contraction. An artificial mould was created using a dental impression material and a full-thickness skin graft. A mould was shaped using $3 \mathrm{M}$ Putty Material and covered with a layer of soft sponge and a condom. Subsequently, the condom was applied, tied on to its open end. The size of the mould corresponding to the neovagina was selected (Supplementary Fig. 4). The fullthickness skin graft was then sewn over this mould with the deep surface of the graft facing outward so as to be in contact with the newly created vaginal wall. The margins were sutured together with synthetic absorbable suture and introduced into the neo-vaginal canal or space (Supplementary Fig. 4). To hold the skin graft covered mould in place for 12 days, the labia were sutured at the midline with interrupted 0 -nylon sutures without tension. A mould covered with a layer of soft sponge and a condom was removed on the 12th postoperative day. The new vagina was thoroughly inspected and irrigated with normal saline solution.

\section{Results}

An unusual Mullerian duct anomaly, uterus bicornis (double uterus) acollis (absence of cervix), was successfully corrected by uteroplasty and a new cervix was constructed. Complete vaginal agenesis in 7 patients was corrected by a modified McIndoe vaginoplasty technique. Clinical examination performed postoperatively revealed a neovagina of adequate length and caliber. None of the 7 patients had any significant post-operative complications.

\section{Discussion}

The genital system is concerned with the maintenance and propagation of the species. In women, the internal genitalia include ovaries, fallopian tubes, the uterus, and the vagina; the external genitalia include mons pubis, labia majora and minora, the clitoris, the vestibule, and the perineum. Gonads initially develop in females (ovaries) and males (testes) from the undifferentiated genital ridge at approximately the 5th week of development, and gonadal differentiation becomes apparent at approximately the 7th week of embryonic life. In embryos of both sexes, the primitive sex ducts are indifferent and consist of 2 paired ducts-mesonephric (Wolffian) and paramesonephric (Mullerian). With the development of the testis from the genital ridge, the mesonephric duct is retained in males as the duct system of the testis and the paramesonephric duct mostly degenerates. In females, however, the paramesonephric duct plays an important role in the development of reproductive organs and the mesonephric duct and its tubules mostly regress.

At first, these paired bilateral Mullerian ducts pass caudally (through the cranial vertical part), lateral to the mesonephric duct. In the pelvis, they cross (through the intermediate horizontal part) ventral to the mesonephric duct and grow medially. During the 8th week, they reach the caudal end of the mesonephric duct and to contact and fuse with their counterparts (caudal vertical part) to form a Y shaped uterovaginal bulb or tubercle that bulges into the dorsal of the urogenital sinus. Caudal vertical parts of both Mullerian ducts fuse in the caudo-cranial direction, which normally occurs between the 6 th and 11 th weeks of gestation. The partition between them completely disappears and forms a single duct uterovaginal canal by the end of 3rd month. The cranial part of the utero-vaginal canal forms the entire uterus, and points of fusion of the 2 ducts represent the site of the future fundus. The cranial vertical parts and most of the intermediate horizontal parts of each Mullerian duct form the respective fallopian tubes. Any disruption of Mullerian duct development and fusion during embryogenesis can result in a broad and complex spectrum of congenital abnormalities, termed Mullerian duct anomalies. A uterus is absent in $2 \%$ to $7 \%$ of pa- 


\section{Obstetrics \& Gynecology Science}

Vol. 63, No. 5, 2020

tients with vaginal agenesis [1]. Congenital anomalies of the uterus result from varying degrees of failure of fusion of the Mullerian ducts but may occasionally arise from true duplication of the ducts [2]. The incidence reported in the literature ranges between $0.13-0.4 \%$ among the general population $[3,4]$. Unilateral suppression of Mullerian ducts results in an unicornuate uterus, which is unique among Mullerian abnormalities and is the rarest of the uterine abnormalities, accounting for $0.3-4 \%$ of uterine abnormalities [5]. Simón et al. [6] also reported that approximately 3.2 percent of fertile women have a bicornuate uterus. In our study (casel), a unique case of an unusual Mullerian duct anomaly, horseshoe shaped uterus bicornis (double uterus) acollis (absence of cervix) along agenesis of the vagina was noted. This type of abnormality may be due to failure of Mullerian duct fusion in the caudo-cranial direction. To the best of our knowledge, such a unique and unusual Mullerian duct anomaly observed in this study has not been cited in modern literature.

The upper four-fifth of the vagina above the hymen develops from the lower part of the utero-vaginal canal and the lower one fifth develops below the hymen from the ectoderm of the genital folds. The vagina opens into the exterior through the vestibule, which is derived from the ectoderm of genital folds after the rupture of the urogenital membrane. Vaginal agenesis (absence of the vagina) is one of the most significant congenital anomalies of the female reproductive tract from a physical and psychological perspective. Vaginal agenesis is estimated to occur in 1 in 4,000-5,000 live female births. The purpose of vaginal agenesis treatment and appropriate management is not only to create an adequate passageway for penetration but also to facilitate satisfactory sexual intercourse and normalize genital anatomy where possible, and to alleviate her psychological concerns. To treat vaginal agenesis, the Mclndoe technique is the preferred method for vaginal reconstruction, and several authors have documented satisfactory sexual relationships using the Mclndoe method in over $75 \%$ of patients [7]. Mayer-RokitanskyKuster Hauser syndrome is a congenital malformation characterized by the failure of the Mullerian duct to develop, resulting vaginal agenesis and absence or hypoplasia of the uterus. Ovaries are normal in the majority of cases, which are more frequently associated with renal and vertebral malformations. Vaginal agenesis is associated with malformations in the urinary tract in $17-56 \%$ of patients [8-10]. In our study, urinary tract malformations were absent. Buss and Lee [11] reported an $80-90 \%$ graft take in $94 \%$ of their patients. Complete graft take 10 days postoperatively was described by Garcia and Jones [12] in 73\% of their patients, with an $80-90 \%$ graft take in a further $20 \%$ of patients. The degree of failure of graft take was not recorded in our present study.

The sex-determining region $Y$ (SRY) gene is located on the short arm of the $Y$ chromosome. During the 6th week of male fetal development, the SRY gene provides instructions for making a protein called the SRY protein (testis determining factor) that influences the development of male phenotypes, such as testes from the genital ridges at the medial posterior abdominal cavity. Complete androgen insensitivity syndrome (testicular feminization) is a syndrome when a male, genetically $X Y$, because of various abnormalities of the $X$ chromosome, is resistant to the actions of androgen hormones, which in turn stops the forming of the male genitalia and gives a female phenotype. This syndrome occurs in one out of 20,000 live births and can be incomplete (various sexual ambiguities) or complete (the person appears to be a woman). Testicular feminization, or androgen insensitivity, is a rare syndrome that is characterized by primary amenorrhea, a 46, XY karyotype, a female phenotype, and the presence of testes rather than ovaries [13-15]. Patients have a congenital resistance to the effects of androgens, which results in an absence of Wolffian duct derivatives and the development of a female phenotype. However, production of the Mullerian inhibitory factor persists, which accounts for the complete regression of the Mullerian duct system. Therefore, patients with testicular feminization have no uterus or fallopian tubes and are without the upper third of the vagina [16]. In approximately one third of these individuals undescended testes have a well-recognized propensity for tumoral proliferation, and the risk of gonadal malignancies increases with age. Hence, it is essential that all women with primary amenorrhea should undergo a complete investigation of the genital tract and endocrine system quickly and accurately to allow clinical and psychologic input.

In conclusion, the findings suggested that a modified McIndoe vaginoplasty technique is a simple, effective procedure for the treatment of vaginal agenesis. We believe that the present study has provided some important data that will contribute to the scientific literature, providing the data of embryogenesis, surgical management, and clinical implications of uterovaginal congenital abnormalities. Awareness of the rare congenital abnormalities noted in our study is essen- 


\section{Obstetrics \& Gynecology Science}

Krishna Gopal Paul, et al. Congenital uterovaginal abnormalities

tial for surgeons, clinical anatomists, radiologists, and morphologists to increase the success of reproductive diagnostic evaluation and surgical approaches to the region.

\section{Conflict of interest}

No potential conflict of interest relevant to this article was reported.

\section{Ethical approval}

This study was reviewed and approved by the appropriate institutional human research ethics committee (reference number: HREC10AUG19).

\section{Patient consent}

Each patient's informed consent for the purpose of this study (i.e. publication without disclosure of personal identity) was obtained.

\section{Supplementary materials}

Supplementary Figures associated with this article can be found online at https://doi.org/10.5468/ogs.2020.20046.

\section{References}

1. Højsgaard A, Villadsen I. McIndoe procedure for congenital vaginal agenesis: complications and results. $\mathrm{Br} J$ Plast Surg 1995;48:97-102.

2. Muckle CW. Developmental abnormalities of the female reproductive organs. In: Sciarra JJ, Dooley S, Depp R, Lurain JR, Kaunitz A, editors. Gynecology and obstetrics looseleaf CD-ROM. Philadelphia (PA): Lippincott Williams
\& Wilkins Publisher; 2001.

3. Golan A, Langer R, Bukovsky I, Caspi E. Congenital anomalies of the müllerian system. Fertil Steril 1989; 51:747-55.

4. Heinonen PK, Saarikoski S, Pystyren P. Reproductive performance of women with uterine anomalies. An evaluation of 182 cases. Acta Obstet Gynecol Scand 982; 61:157-62.

5. Heinonen PK. Unicornuate uterus and rudimentary horn. Fertil Steril 1997;68:224-30.

6. Simón C, Martinez L, Pardo F, Tortajada M, Pellicer A. Müllerian defects in women with normal reproductive outcome. Fertil Steril 1991;56:1192-3.

7. Tolhurst $D E$, van der Helm TW. The treatment of vaginal atresia. Surg Gynecol Obstet 1991;172:407-14.

8. Fliegner JR. Congenital atresia of the vagina. Surg Gynecol Obstet 1987;165:387-91.

9. Salvatore CA, Lodovicci O. Vaginal agenesis: an analysis of ninety cases. Acta Obstet Gynecol Scand 1978; 57:89-94.

10. Fore SR, Hammond CB, Parker RT, Anderson EE. Urologic and genital anomalies in patients with congenital absence of the vagina. Obstet Gynecol 1975;46:410-6.

11. Buss JG, Lee RA. Mclndoe procedure for vaginal agenesis: results and complications. Mayo Clin Proc 1989; 64:758-61.

12. Garcia J, Jones HW Jr. The split thickness graft technic for vaginal agenesis. Obstet Gynecol 1977;49:328-32.

13. Coulam CB, Graham ML 2nd, Spelsberg TC. Androgen insensitivity syndrome: gonadal androgen receptor activity. Am J Obstet Gynecol 1984;150:531-3.

14. Rutgers JL, Scully RE. The androgen insensitivity syndrome (testicular feminization): a clinicopathologic study of 43 cases. Int J Gynecol Pathol 1991;10:126-44.

15. Griffin JE, Wilson JD. The syndromes of androgen resistance. N Engl J Med 1980;302:198-209.

16. Gîngu $C$, Dick $A$, Pătrăşcoiu $S$, Domnişor $L$, Mihai $M$, Hârza $M$, et al. Testicular feminization: complete androgen insensitivity syndrome. Discussions based on a case report. Rom J Morphol Embryol 2014;55:177-81. 